\title{
PROPUESTA DE INNOVACIÓN METODOLÓGICA EN LA ENSEÑANZA SUPERIOR: FLIPPED CLASSROOM, PRÁCTICA REFLEXIVA Y EVALUACIÓN FORMATIVA
}

A proposal of methodological innovation in higher education: flipped classroom, reflective practice and formative evaluation

Proposta inovação metodológica no ensino superior: Flipped classroom, prática reflexiva e avaliação formativa

\section{Inmaculada Fuentes-García (1)}

Fernando M. Otero-Saborido (2)

\section{Antonio Sánchez-Oliver (3)}

(1) Universidad Pablo de Olavide de Sevilla, España. Correo electrónico: ifuegar@upo.es

(2) Universidad Pablo de Olavide de Sevilla, España. Teléfono: 954977 961. Correo electrónico: fmotero@upo.es

(3) Universidad Pablo de Olavide de Sevilla, España. Correo electrónico: asanchez@upo.es

\section{Resumen}

Actualmente se ha demostrado que el aprendizaje es fruto de lo que el alumno hace y no tanto de lo que hace el profesor. La creciente incorporación de metodologías activas en la enseñanza pretende desplazar a la pedagogía tradicional, convirtiendo al alumnado en (verdadero) protagonista activo de su proceso de enseñanza- aprendizaje. El objetivo de este trabajo es presentar una propuesta de innovación metodológica para la enseñanza superior en la que la acción pedagógica del profesor se enfoque como una orientación dentro de un proceso significativo y constructivo donde el trabajo del alumno tome protagonismo. De esta forma se minimizará el actual déficit entre créditos ECTS y horas reales, en consonancia con las pretensiones del sistema acumulado de transferencia de créditos y nos aproximaremos a una adquisición real de competencias y aprendizajes funcionales por parte del alumnado.

Propuesta de innovación metodológica en la enseñanza superior: flipped classroom, práctica reflexiva y evaluación formativa 
Palabras clave: Flipped classroom; práctica reflexiva; evaluación formativa; competencias

\begin{abstract}
Nowadays it has been demonstrated that the learning is result of what the student does and not so much of what the teacher does. The increasing incorporation of active methodologies in teaching aims to displace the traditional pedagogy, turning students into (true) active protagonist of their teaching-learning process. The purpose of this work is to present a proposal of methodological innovation for higher education in which the teacher's pedagogical action is focused as an orientation within a meaningful and constructive process where the work of the student work takes prominence. In this way, the current deficit between ECTS credit and actual hours will be minimized in line with the pretensions of the European Credit Transfer and Accumulation System and we will be close to a real acquisition of competences and functional learning by the students.
\end{abstract}

Keywords: Flipped classroom; Reflexive practice; Formative assessment; Competencies

\title{
Resumo
}

Hoje em dia tem sido demonstrado que o aprendizado é resultado do que o aluno faz e não tanto do que o professor faz. A crescente incorporação de metodologias ativas no ensino tem como objetivo deslocar a pedagogia tradicional, transformando os alunos em verdadeira protagonista ativa de seu processo de ensino-aprendizagem. O objetivo deste trabalho é apresentar uma proposta de inovação metodológica para o ensino superior, na qual a ação pedagógica do professor é focalizada como uma orientação dentro de um processo construtivo e significativo onde o trabalho do trabalho do aluno ganha destaque. Desta forma, o déficit atual entre o crédito ECTS e as horas efetivas será minimizado de acordo com as pretensões do Sistema Europeu de Transferência e Acumulação de Créditos e estaremos perto de uma verdadeira aquisição de competências e aprendizagem funcional pelos alunos.

Palavras-chave: Flipped classroom; prática reflexiva; avaliaçãoformativa; competências

Propuesta de innovación metodológica en la enseñanza superior: flipped classroom, práctica reflexiva y evaluación formativa 


\section{Introducción}

Los resultados de la investigación educativa han demostrado que la clave del éxito del proceso de enseñanza-aprendizaje es facilitar las condiciones necesarias para que los estudiantes aprendan por sí mismos. A pesar de ello, en la mayoría de las aulas continúa imperando la pedagogía tradicional, centrada en la enseñanza y el docente más que en el aprendizaje y el discente.

En la enseñanza superior con la implantación del Sistema Acumulado de Transferencia de Créditos se empieza a apostar por una nueva metodología que sitúa al alumno en el centro del proceso de enseñanza-aprendizaje. Se pretende cuantificar tanto las horas de docencia como el trabajo no presencial del alumno, incluyendo el tiempo de preparación de pruebas de evaluación, lecturas, seminarios, etc.

En diferentes foros educativos se habla de "la ficción de los ECTS" como una deficiencia entre los créditos otorgados a una asignatura y la carga crediticia que desarrolla el alumno fuera de las horas no lectivas (Gómez, et al., 2011; Prieto, Díaz, Monserrat, \& Reyes, 2014). Este déficit entre ECTS y horas reales no es sólo una cuestión cuantitativa, sino que supone un problema de mayores dimensiones al poner en entredicho la perseguida adquisición de competencias y aprendizajes funcionales por parte del alumnado.

Basándonos en una metodología participativa, presentamos una propuesta de innovación docente que va más allá de la exclusiva acumulación de conocimientos por parte del alumnado y que persigue la práctica de habilidades específicas que contribuirán al desarrollo de las competencias genéricas.

\section{Contextualización}

La propuesta se llevará a cabo en la asignatura de Fundamentos de la Educación Física y del Deporte, de $1^{0}$ de Grado de Ciencias de la Actividad Física y del Deporte de la Universidad Pablo de Olavide.

La carga crediticia de la asignatura es de 6 créditos ECTS, equivalentes a 150 horas de trabajo del alumnado y el número de matriculados previsto de 140 alumnos.

Propuesta de innovación metodológica en la enseñanza superior: flipped classroom, práctica reflexiva y evaluación formativa 


\section{Diseño y desarrollo}

En el desarrollo de la propuesta pueden distinguirse dos momentos: la primera semana de clase se dedicará a la explicación de la metodología de trabajo y a la familiarización del alumno con la misma. A partir de la segunda semana de clase comenzará a implantarse la dinámica de trabajo innovador con un patrón de trabajo muy parecido durante todo el curso (figura 1).

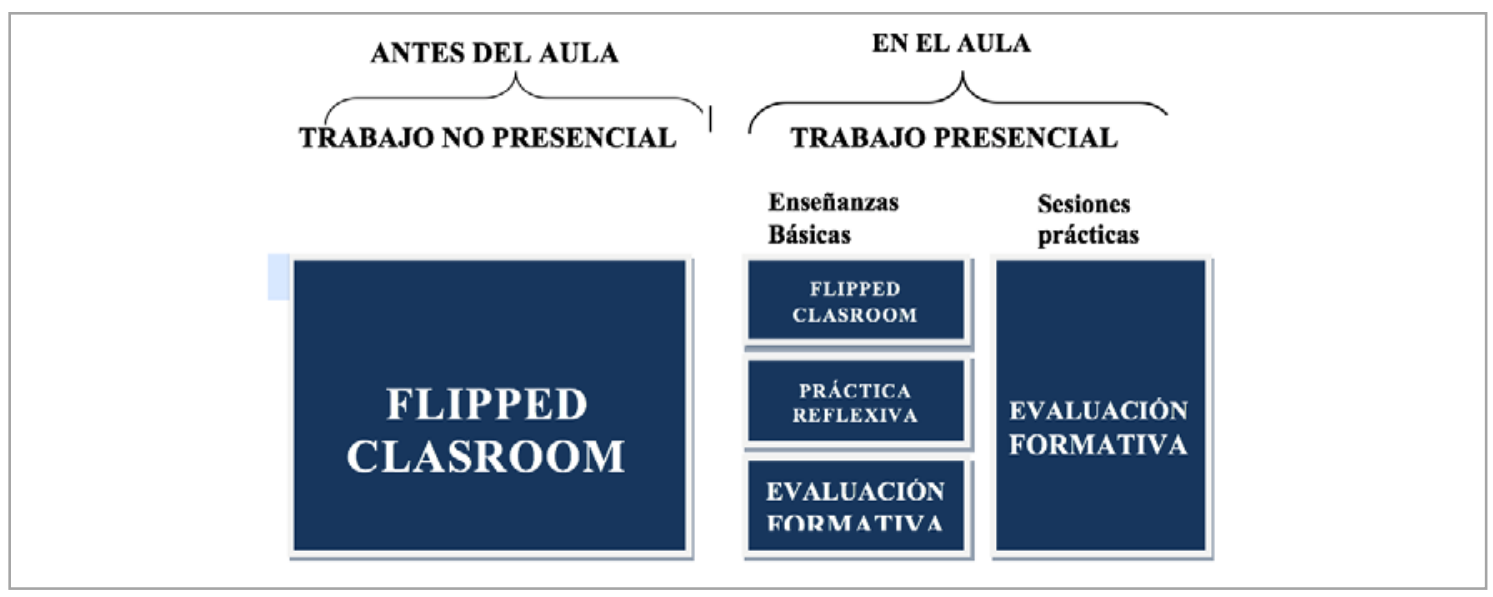

Figura 1.

Organización de las estrategias metodológicas durante la aplicación de la propuesta de innovación.

La propuesta se desarrollará a través de las siguientes estrategias metodológicas: flipped classroom o aula invertida, práctica reflexiva y evaluación formativa (figura 2).

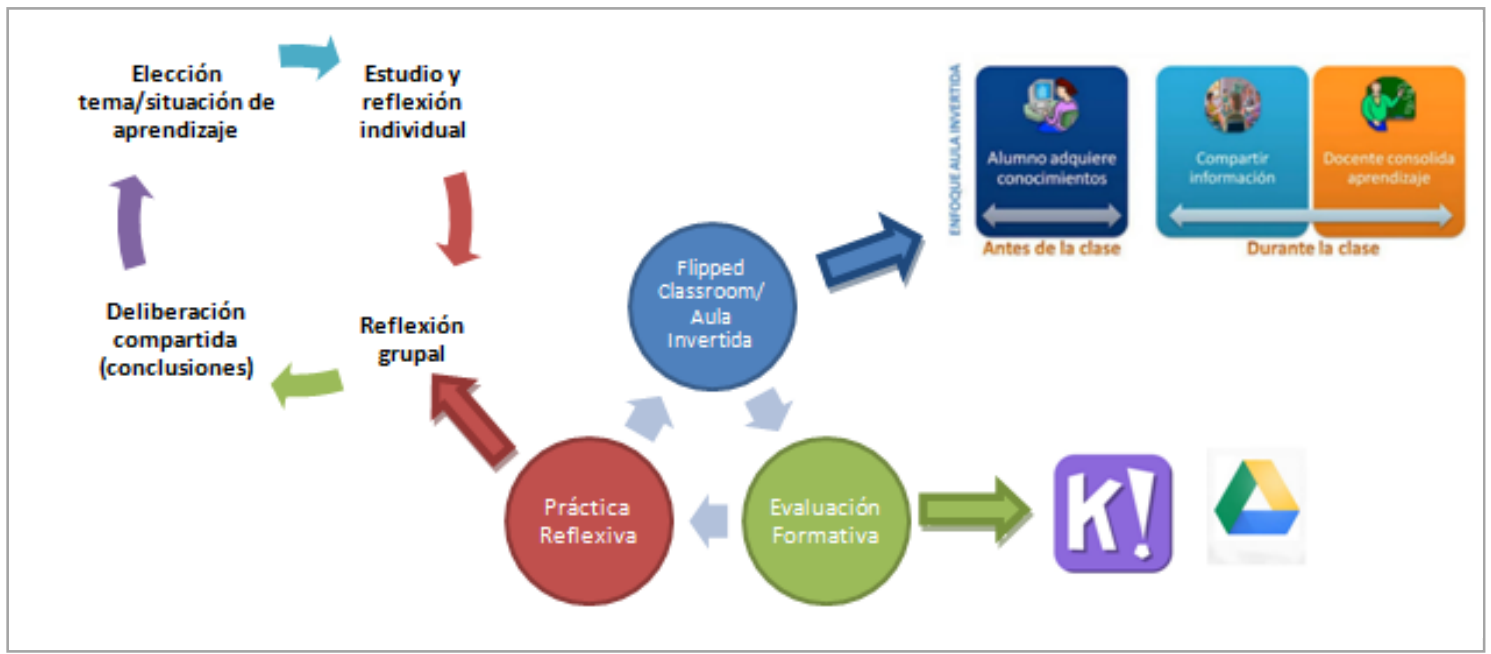

Figura 2.

Estrategias metodológicas para el desarrollo de la propuesta de innovación docente: FlippedClassroom, práctica reflexiva y evaluación formativa.

Propuesta de innovación metodológica en la enseñanza superior: flipped classroom, práctica reflexiva y evaluación formativa 
Flipped Classroom (FC) o Aula Invertida es un modelo pedagógico que se basa en la inversión de la estructura tradicional de la clase presencial expositiva a través del empleo de tecnologías de información y comunicación. Antes de la clase produciremos o seleccionaremos material digital (videos, documentos y artículos, infografías, páginas webs, presentación audiovisual, etc.) donde se expondrán los diferentes contenidos de la asignatura. El alumno trabajará antes de la clase de forma autónoma y responderá, a través de cuestionarios de Google drive, una serie de preguntas de comprensión del tema expuesto en los videos del "site” de la asignatura. Acudirá al aula familiarizado con el contenido a tratar y el profesor, en lugar de centrarse en la exposición teórica, aclarará los conceptos más complejos, asistirá de forma individual a los alumnos con dificultades y fomentará el compromiso de los estudiantes con su propio aprendizaje (O'Flaherty \& Phillips, 2015; McLean, Attardi, Faden \& Goldszmidt, 2016).

La Práctica Reflexiva es una herramienta de aprendizaje que permite al discente implicarse y analizar un tema. El alumnado deberá asistir a las sesiones teóricas y/o prácticas con una reflexión individual escrita sobre el tema propuesto. Trabajarán los temas antes de su llegada al aula para posteriormente en la clase realizar una reflexión grupal que finalizará con la exposición de conclusiones al resto de compañeros (figura 2).

La Evaluación Formativa debe ser entendida como sinónimo de aprendizaje y no exclusivamente de calificación. Durante las clases se recurrirá a herramientas como "Kahoot” para situaciones de evaluación por pares y grupales; los alumnos se enfrentarán a exposiciones individuales y en grupo sobre los contendidos trabajados para posteriormente llevar a cabo procesos de diálogo profesor-alumno y alumnoalumno. Al finalizar la semana, cada alumno realizará una autoevaluación de su implicación en los módulos desarrollados y calculará su implicación horaria para determinar la relación trabajo real-ECTS.

\section{Evaluación}

Los procesos de evaluación constituyen una herramienta metodológica clave para lograr el objetivo de nuestra propuesta. La calificación del alumno en la asignatura no será más que el resultado de todo unproceso de evaluación continua y formativa. Las estrategias de evaluación formativa a utilizar desde el punto de vista de la participación del alumno Propuesta de innovación metodológica en la enseñanza superior: flipped classroom, práctica reflexiva y evaluación formativa 
son la autoevaluación (evaluación que el alumno realizará sobre sí mismo), la coevaluación (evaluación entre iguales), evaluación compartida (procesos de diálogo que mantiene el profesorado con su alumnado sobre la evaluación de los aprendizajes) y evaluación democrática (concretamente participarán en la elección de algunas de las preguntas la prueba final escrita).

El desglose de la calificación se detalla en la tabla 1.

Tabla 1.

Puntuación asignada a los aspectos evaluados para el cálculo de la calificación final.

\begin{tabular}{lcc}
\multicolumn{1}{c}{ Aspectos/ítems a evaluar } & $\begin{array}{c}\text { \% de la } \\
\text { calificación }\end{array}$ & $\begin{array}{c}\text { Puntuación para la } \\
\text { calificación. }\end{array}$ \\
\hline $\begin{array}{l}\text { Implicación activa del alumno (visionado vídeos, lecturas, } \\
\text { reflexiones, etc). }\end{array}$ & 50 & 5 puntos \\
$\begin{array}{l}\text { Procesos de evaluación formativa (autoevaluaciones, } \\
\text { evaluación recíproca, evaluación compartida, reflexión sobre el } \\
\text { grado de implicación). }\end{array}$ & 10 & 1 punto \\
Prueba final escrita. & 40 & 4 puntos \\
\hline
\end{tabular}

\section{Conclusiones}

La iniciativa da respuesta a "la ficción de los ECTS". Las estrategias utilizadas deben entenderse como herramientas dentro del conjunto de metodologías activas que ponen al alumnado en el centro del aprendizaje. Estos enfoques redefinen los roles de los protagonistas del proceso. Los docentes dejan de ser meros transmisores para adquirir funciones de mediadores, dinamizadores y diseñadores de ambientes de aprendizajes. El alumnado abandona el rol de receptor pasivo y se empodera como constructor de su aprendizaje.

\section{Referencias}

Arrobas, T., Cazenave, J., Cañizares, J., \& Fernández, M. (2016). Herramientas didácticas para mejorar el rendimiento académico. REDU. Revista de Docencia Universitaria, 12(4), 397-413.

Gómez, F.; Barjola, P. Díaz, G., González, J.L., López, A., Mercado, F.J., \&Rivas, I. (2011). Cálculo del volumen de trabajo del alumno en las nuevas titulaciones de ciencias de la salud. En F. Labrador \& R. Santero. (Ed.), Evaluación global de los resultados del aprendizaje en las titulaciones dentro del Espacio Europeo de Educación Superior (pp. 417-436). Madrid: Dykinson.

Propuesta de innovación metodológica en la enseñanza superior: flipped classroom, práctica reflexiva y evaluación formativa 
Herreid, C. F., \& Schiller, N. A. (2013). Case studies and the flipped classroom. Journal of College Science Teaching, 42(5), 62-66.

López, M.A. (2005). La participación del alumnado en la evaluación: la autoevaluación, la coevaluación y la evaluación compartida. Tándem, 17, 21-37

McLean, S., Attardi, S. M., Faden, L., \& Goldszmidt, M. (2016). Flipped classrooms and student learning: not just surface gains. Advances in Physiology Education, 40(1), 47-55.

Martínez, F. (2009). Evaluación formativa en aula y evaluación a gran escala: hacia un sistema más equilibrado. Revista electrónica de investigación educativa, 11(2), 118.

O'Flaherty, J., y Phillips, C. (2015). The use of flipped classrooms in higher education: A scoping review. The Internet and Higher Education, 25, 85-95.

Prieto Martín, A., Díaz Martin, D., Monserrat Sanz, J., y Reyes Martín, E. (2014). Experiencias de aplicación de estrategias de gamificación a entornos de aprendizaje universitario. ReVision, 7(2), 76-92.

Talbert, R. (2014). Inverted classroom. Colleagues, 9(1),Article 7. Recuperado de: http://scholarworks.gvsu.edu/colleagues/vol9/iss1/7

Tucker, B. (2012). The flipped classroom. Education Next, 12 (1), 82-83. 